\title{
Influence of Different Onion Cultivars on Storage Life under Hill Zone of Karnataka
}

\author{
Veena Goni", Devaraju, V. Srinivasa, D. Lakshmana and Y. Kantharaj \\ Department of Vegetable Science, College of Horticulture, Mudigere, University of \\ Agricultural and Horticultural sciences, Shivamogga, Karnataka, India \\ *Corresponding author
}

\section{A B S T R A C T}

\section{Keywords}

Onion cultivars, Storage life, Sprouting

Article Info

Accepted: 24 September 2020 Available Online: 10 October 2020
An experiment was conducted to study the storage behavior among thirty onion genotypes under ambient condition for four months. The experiment results revealed that after four months of storage, the minimum per cent of sprouting was noticed in Bhima Shakti (7.68 \%), while, the maximum per cent of sprouting was noticed in N-53 (15.10\%). The minimum per cent of rotting was observed in Bhima Shakti (7.34 \%) and the maximum per cent of rotting was recorded in N-53 $(14.78 \%)$.With respect to total loss in weight, the cultivar Bhima Shakti (16.05 $\%)$ recorded minimum loss in weight after 4 months of storage, while, Poona Nasangi $(28.63 \%)$ recorded maximum loss in weight.

\section{Introduction}

Onion is one of the most important vegetable crops commercially grown in the world.It is a diploid $(2 \mathrm{n}=2 \mathrm{x}=16)$, herbaceous biennial in the Amaryllidaceae family and is one of the monocotyledonous crops. It belongs to the genus Allium which consists of about 750 species, among which, Japanese bunching onion, leeks, and garlic are the most important edible Allium crops (Rabinowitch and Currah, 2002). It is probably originated from Central Asia between Turkmenistan and Afghanistan where some of its relatives are still growing in the wild forms.
The Mediterranean regions are considered to be the secondary centre of origin (CSIR, 2003). Onion is a short duration horticultural crop (Brewster, 1990) grown at low latitudes. It is semi-perishable in nature and gets deteriorated during storage, transportation and marketing. Bulbs stored under ambient conditions are more prone to storage losses. These losses are comprised of physiological loss in weight i.e., moisture losses and shrinkage (30-40\%), rotting (10-12\%) and sprouting (8-10\%). Factors such as high respiration rate, biochemical changes, physiological injuries, water loss and physiological disorders are mainly responsible for post-harvest losses of onion 
bulbs (Kedar et al., 1989). Increase in temperature increases the sprouting per cent of onion bulbs, while, increase in humidity increases the rotting per cent of bulbs. Despite the achievements in production technology, post-harvest losses during storage still pose a great problem. Hence, it becomes necessary to study the performance of onion genotypes for storage characteristics.

\section{Materials and Methods}

The experiment was conducted at the experimental block of Department of Vegetable Science, College of Horticulture, Mudigere, which is situated in the hill zone of Karnataka during Rabi season. The study was conducted using Randomized Complete Block Design (RCBD) with two replications involving thirty genotypes which were procured from various research centres (Table 1). The bulbs were harvested and field cured for a week. Four $\mathrm{kg}$ of bulbs were selected randomly from each replication and the initial number of bulbs of all the cultivars per $4 \mathrm{~kg}$ was recorded replication wise. The bulbs were stored for 4 months under ambient conditions. Observations on storage characters viz., per cent loss due to sprouting, per cent loss due to rotting and total loss in weight were recorded.

The per cent loss due to sprouting was calculated by weighing the bulbs that were sprouted in different treatments and the per cent loss due to rotting was calculated by weighing the bulbs that were rotten in different treatments. The weight of bulbs prior to storage and weight after storage were recorded at 15 days interval for 4 months and the difference was worked out to estimate the total loss in weight.

\section{Results and Discussion}

Results pertaining to per cent loss due to sprouting, per cent loss due to rotting and total loss in weight among thirty onion genotypes is depicted in Table 2 and 3 .

In the present investigation, the per cent loss due to sprouting was not noticed in any of the genotypes during first and second months after storage. However, during third month of storage, per cent loss due to sprouting was maximum in PusaRed (4.37\%), whereas, minimum in Bhima Shubra (1.21\%). During fourth month of storage, it was maximum in $\mathrm{N}-53(15.10 \%)$ and minimum in Bhima Shakti (7.68 \%). Similar findings were reported by Shanmugasundaram (1999), Trivedi and Dhumal (2010) and Utagi et al., (2015). Brewster (1994) reported that factors such as stage of bulb development during harvest, premature defoliation, skin integrity, harvesting, conditions during ripening and curing are responsible for sprouting.

The results regarding per cent loss due to rotten bulbs revealed that, during first month after storage, none of the genotypes exhibited rotting. However, during second, third and fourth month after storage, significant difference was observed for per cent loss due to rotten bulbs. Cultivar N-53 recorded highest per cent of rotten bulbs during second and fourth month after storage $(4.87 \%$ and $14.78 \%$ respectively), while, $\mathrm{W}-125(4.38 \%)$ during third month after storage. The minimum per cent of rotten bulbs was observed in Arka Niketan (1.27\%), W-405 (1.06 \%) and Bhima Shakti (7.34 \%) during second, third and fourth month after storage respectively. Shanmugasundaram (1999), Shanmugasundaram (2000), Trivedi and Dhumal (2010), Dhotre (2009), also observed significant difference with respect to per cent loss due to rotting among different genotypes of onion. Kukanoor (2005) reported that due to high moisture content on outer scales, the onion bulbs are more prone to rotting loss after harvest. 
The genotypes exhibited significant difference in respect of total loss in weight. The total loss in weight was maximum in $\mathrm{N}$ 53 (5.38 \%), Bhima Red (12.27 \%), Bhima Super $(21.37 \%)$ and Poona Nasangi (28.63 $\%$ ) after first, second, third and fourth month after storage respectively, while it was minimum in Bhima Kiran $(1.07 \%)$ and Bhima Super $(7.45 \%)$ after first and second month after storage respectively. During third and fourth month after storage, it was minimum in Bhima Shakti $(10.33 \%$ and $16.05 \%$ respectively). The results are in collaboration with findings of Shanmugasundaram (2000), Shanmugasundaram (2003), Trivedi and Dhumal (2010), Jamali et al., (2012).

Table.1 List of onion genotypes used in the study and their sources of collection

\begin{tabular}{|c|c|c|}
\hline SI.No & Genotypes & Source of collection \\
\hline 1. & Arka Kalyan & IIHR, Bengaluru \\
\hline 2. & Arka Niketan & IIHR, Bengaluru \\
\hline 3. & Bhima Dark Red & DOGR, Pune \\
\hline 4. & Bhima Kiran & DOGR, Pune \\
\hline 5. & Bhima Light Red & DOGR, Pune \\
\hline 6. & Bhima Raj & DOGR, Pune \\
\hline 7. & Bhima Red & DOGR, Pune \\
\hline 8. & Bhima Safed & DOGR, Pune \\
\hline 9. & Bhima Shakti & DOGR, Pune \\
\hline 10. & Bhima Shubhra & DOGR, Pune \\
\hline 11. & Bhima Super & DOGR, Pune \\
\hline 12. & Bhima Swetha & DOGR, Pune \\
\hline 13. & $\mathrm{~N}-53$ & DOGR, Pune \\
\hline 14. & Poona Nasangi & Local collection \\
\hline 15. & Pusa Red & IARI, New Delhi \\
\hline 16. & W - 045 & DOGR, Pune \\
\hline 17. & $\mathrm{~W}-125$ & DOGR, Pune \\
\hline 18. & W - 143 & DOGR, Pune \\
\hline 19. & W - 177 & DOGR, Pune \\
\hline 20. & W - 182 & DOGR, Pune \\
\hline 21. & W - 203 & DOGR, Pune \\
\hline 22. & W - 210 & DOGR, Pune \\
\hline 23. & W - 226 & DOGR, Pune \\
\hline 24. & W - 253 & DOGR, Pune \\
\hline 25. & W - 364 & DOGR, Pune \\
\hline 26. & W - 405 & DOGR, Pune \\
\hline 27. & W - 444 & DOGR, Pune \\
\hline 28. & W - 464 & DOGR, Pune \\
\hline 29. & W - 498 & DOGR, Pune \\
\hline 30. & W - 500 & DOGR, Pune \\
\hline
\end{tabular}


Table.2 Storage behavior of onion bulbs among thirty genotypes of onion (After 1 and 2 months of storage)

\begin{tabular}{|c|c|c|c|c|c|c|c|c|c|}
\hline \multirow{2}{*}{$\begin{array}{l}\text { Sl. } \\
\text { No }\end{array}$} & \multirow[t]{2}{*}{ Genotypes } & \multirow{2}{*}{$\begin{array}{c}\text { Initial } \\
\text { weight of } \\
\text { bulbs }(\mathrm{kg})\end{array}$} & \multirow{2}{*}{$\begin{array}{c}\text { Total } \\
\text { number of } \\
\text { bulbs }\end{array}$} & \multicolumn{3}{|c|}{ Per cent loss one month after storage } & \multicolumn{3}{|c|}{ Per cent loss two month after storage } \\
\hline & & & & $\begin{array}{c}\text { Per cent of } \\
\text { sprouting } \\
\text { bulbs }\end{array}$ & $\begin{array}{l}\text { Per cent of } \\
\text { rotten bulbs }\end{array}$ & $\begin{array}{l}\text { Total loss in } \\
\text { weight }(\%)\end{array}$ & $\begin{array}{l}\text { Per cent of } \\
\text { sprouting } \\
\text { bulbs }\end{array}$ & $\begin{array}{l}\text { Per cent of } \\
\text { rotten bulbs }\end{array}$ & $\begin{array}{c}\text { Total loss in } \\
\text { weight }(\%)\end{array}$ \\
\hline 1. & Arka Kalyan & 4.00 & 44 & 0 & 0 & 4.33 & 0 & 2.83 & 10.08 \\
\hline 2. & Arka Niketan & 4.00 & 42 & 0 & 0 & 3.25 & 0 & 1.27 & 9.23 \\
\hline 3. & Bhima Dark Red & 4.00 & 40 & 0 & 0 & 1.89 & 0 & 2.45 & 10.33 \\
\hline 4. & Bhima Kiran & 4.00 & 45 & 0 & 0 & 1.07 & 0 & 2.57 & 8.57 \\
\hline 5. & Bhima Light Red & 4.00 & 51 & 0 & 0 & 3.02 & 0 & 3.02 & 11.25 \\
\hline 6. & Bhima Raj & 4.00 & 48 & 0 & 0 & 2.68 & 0 & 2.35 & 7.55 \\
\hline 7. & Bhima Red & 4.00 & 43 & 0 & 0 & 1.08 & 0 & 1.75 & 12.27 \\
\hline 8. & Bhima Safed & 4.00 & 56 & 0 & 0 & 4.13 & 0 & 2.71 & 7.78 \\
\hline 9. & Bhima Shakti & 4.00 & 42 & 0 & 0 & 2.74 & 0 & 1.41 & 11.56 \\
\hline 10. & Bhima Shubhra & 4.00 & 49 & 0 & 0 & 2.33 & 0 & 1.52 & 10.16 \\
\hline 11. & Bhima Super & 4.00 & 45 & 0 & 0 & 3.63 & 0 & 3.09 & 7.45 \\
\hline 12. & Bhima Swetha & 4.00 & 46 & 0 & 0 & 2.13 & 0 & 2.18 & 8.41 \\
\hline 13. & $\mathrm{~N}-53$ & 4.00 & 44 & 0 & 0 & 5.38 & 0 & 4.87 & 10.25 \\
\hline 14. & Poona Nasangi & 4.00 & 50 & 0 & 0 & 2.98 & 0 & 1.34 & 11.34 \\
\hline 15. & Pusa Red & 4.00 & 52 & 0 & 0 & 2.29 & 0 & 1.32 & 8.12 \\
\hline 16. & W - 045 & 4.00 & 47 & 0 & 0 & 2.31 & 0 & 3.08 & 8.62 \\
\hline 17. & W - 125 & 4.00 & 45 & 0 & 0 & 2.56 & 0 & 1.68 & 12.13 \\
\hline 18. & W - 143 & 4.00 & 46 & 0 & 0 & 2.34 & 0 & 3.34 & 7.81 \\
\hline 19. & W - 177 & 4.00 & 54 & 0 & 0 & 3.35 & 0 & 2.11 & 10.62 \\
\hline 20. & W - 182 & 4.00 & 50 & 0 & 0 & 3.89 & 0 & 1.79 & 10.06 \\
\hline 21. & W - 203 & 4.00 & 49 & 0 & 0 & 2.66 & 0 & 2.08 & 7.67 \\
\hline 22. & W - 210 & 4.00 & 54 & 0 & 0 & 2.31 & 0 & 3.14 & 11.73 \\
\hline 23. & W - 226 & 4.00 & 51 & 0 & 0 & 1.71 & 0 & 1.47 & 11.14 \\
\hline 24. & W - 253 & 4.00 & 48 & 0 & 0 & 1.41 & 0 & 3.98 & 8.64 \\
\hline 25. & W - 364 & 4.00 & 52 & 0 & 0 & 2.63 & 0 & 2.26 & 7.76 \\
\hline 26. & W - 405 & 4.00 & 45 & 0 & 0 & 2.61 & 0 & 2.87 & 10.98 \\
\hline 27. & W - 444 & 4.00 & 49 & 0 & 0 & 3.21 & 0 & 2.23 & 12.07 \\
\hline 28. & W - 464 & 4.00 & 51 & 0 & 0 & 2.50 & 0 & 2.72 & 8.83 \\
\hline 29. & W - 498 & 4.00 & 51 & 0 & 0 & 3.11 & 0 & 3.18 & 8.43 \\
\hline \multirow[t]{4}{*}{30.} & W - 500 & 4.00 & 50 & 0 & 0 & 2.93 & 0 & 1.58 & 10.34 \\
\hline & Mean & - & - & 0 & 0 & 2.75 & 0 & 2.41 & 9.71 \\
\hline & S.Em \pm & - & - & - & - & 0.05 & - & 0.03 & 0.02 \\
\hline & C.D@ 5\% & - & - & - & - & 0.15 & - & 0.09 & 0.06 \\
\hline
\end{tabular}


Table.3 Storage behavior of onion bulbs among thirty genotypes of onion (After 3 and 4 months of storage)

\begin{tabular}{|c|c|c|c|c|c|c|c|c|c|}
\hline \multirow{2}{*}{$\begin{array}{l}\text { Sl. } \\
\text { No }\end{array}$} & \multirow[t]{2}{*}{ Genotypes } & \multirow{2}{*}{$\begin{array}{c}\text { Initial } \\
\text { weight of } \\
\text { bulbs (kg) }\end{array}$} & \multirow{2}{*}{$\begin{array}{c}\text { Total } \\
\text { number of } \\
\text { bulbs }\end{array}$} & \multicolumn{3}{|c|}{ Per cent loss three month after storage } & \multicolumn{3}{|c|}{ Per cent loss four month after storage } \\
\hline & & & & $\begin{array}{l}\text { Per cent of } \\
\text { sprouting } \\
\text { bulbs }\end{array}$ & $\begin{array}{l}\text { Per cent of } \\
\text { rotten bulbs }\end{array}$ & $\begin{array}{l}\text { Total loss in } \\
\text { weight }(\%)\end{array}$ & $\begin{array}{c}\text { Per cent of } \\
\text { sprouting } \\
\text { bulbs }\end{array}$ & $\begin{array}{l}\text { Per cent of } \\
\text { rotten bulbs }\end{array}$ & $\begin{array}{l}\text { Total loss in } \\
\text { weight }(\%)\end{array}$ \\
\hline 1. & Arka Kalyan & 4.00 & 44 & 1.41 & 2.62 & 10.70 & 12.70 & 13.05 & 20.37 \\
\hline 2. & Arka Niketan & 4.00 & 42 & 2.23 & 2.47 & 17.43 & 10.00 & 9.33 & 24.22 \\
\hline 3. & Bhima Dark Red & 4.00 & 40 & 2.24 & 3.08 & 15.44 & 10.44 & 8.87 & 18.40 \\
\hline 4. & Bhima Kiran & 4.00 & 45 & 3.13 & 4.37 & 12.73 & 7.70 & 9.35 & 17.50 \\
\hline 5. & Bhima Light Red & 4.00 & 51 & 4.05 & 2.10 & 18.45 & 9.65 & 11.19 & 21.09 \\
\hline 6. & Bhima Raj & 4.00 & 48 & 1.60 & 1.23 & 16.11 & 12.35 & 10.39 & 18.57 \\
\hline 7. & Bhima Red & 4.00 & 52 & 4.37 & 2.32 & 17.77 & 12.21 & 10.48 & 22.61 \\
\hline 8. & Bhima Safed & 4.00 & 56 & 1.33 & 4.21 & 16.09 & 11.31 & 12.54 & 20.57 \\
\hline 9. & Bhima Shakti & 4.00 & 42 & 2.95 & 1.23 & 10.33 & 7.68 & 7.34 & 16.05 \\
\hline 10. & Bhima Shubhra & 4.00 & 49 & 1.21 & 3.04 & 18.62 & 11.52 & 12.40 & 22.63 \\
\hline 11. & Bhima Super & 4.00 & 45 & 1.79 & 3.58 & 21.37 & 11.06 & 9.31 & 21.46 \\
\hline 12. & Bhima Swetha & 4.00 & 46 & 3.11 & 3.75 & 17.01 & 10.78 & 11.24 & 24.01 \\
\hline 13. & $\mathrm{~N}-53$ & 4.00 & 44 & 2.48 & 2.33 & 12.84 & 15.10 & 14.78 & 23.35 \\
\hline 14. & Poona Nasangi & 4.00 & 50 & 1.36 & 4.03 & 18.31 & 9.44 & 11.66 & 28.63 \\
\hline 15. & Pusa Red & 4.00 & 52 & 4.37 & 2.32 & 17.77 & 12.21 & 10.48 & 22.61 \\
\hline 16. & W - 045 & 4.00 & 47 & 2.96 & 2.66 & 15.07 & 12.33 & 10.65 & 26.66 \\
\hline 17. & W - 125 & 4.00 & 45 & 1.70 & 4.38 & 12.63 & 9.65 & 10.77 & 19.50 \\
\hline 18. & W -143 & 4.00 & 46 & 3.91 & 2.69 & 19.35 & 9.87 & 7.42 & 18.43 \\
\hline 19. & W - 177 & 4.00 & 54 & 2.53 & 1.57 & 18.44 & 13.68 & 12.41 & 17.34 \\
\hline 20. & W - 182 & 4.00 & 50 & 2.78 & 3.41 & 17.32 & 10.76 & 8.75 & 24.81 \\
\hline 21. & W - 203 & 4.00 & 49 & 3.09 & 3.30 & 15.39 & 11.54 & 13.15 & 21.49 \\
\hline 22. & W - 210 & 4.00 & 54 & 2.61 & 2.36 & 16.21 & 10.25 & 9.60 & 25.64 \\
\hline 23. & W - 226 & 4.00 & 51 & 1.57 & 3.57 & 10.48 & 14.10 & 12.64 & 24.46 \\
\hline 24. & W - 253 & 4.00 & 48 & 4.35 & 3.22 & 14.73 & 14.03 & 12.26 & 20.86 \\
\hline 25. & W - 364 & 4.00 & 52 & 2.51 & 2.97 & 18.69 & 12.51 & 10.24 & 23.39 \\
\hline 26. & W - 405 & 4.00 & 45 & 3.12 & 1.06 & 20.05 & 10.98 & 12.37 & 21.09 \\
\hline 27. & W - 444 & 4.00 & 49 & 2.68 & 1.77 & 11.46 & 12.24 & 10.86 & 17.23 \\
\hline 28. & W - 464 & 4.00 & 51 & 1.74 & 3.65 & 15.32 & 11.63 & 9.23 & 22.69 \\
\hline 29. & W - 498 & 4.00 & 51 & 1.58 & 2.86 & 15.21 & 10.86 & 13.63 & 19.86 \\
\hline \multirow[t]{4}{*}{30.} & W - 500 & 4.00 & 50 & 3.07 & 2.75 & 20.27 & 13.34 & 8.81 & 20.21 \\
\hline & Mean & - & - & 2.54 & 2.85 & 15.82 & 11.43 & 10.90 & 21.62 \\
\hline & S.Em \pm & - & - & 0.03 & 0.05 & 0.13 & 0.08 & 0.09 & 1.92 \\
\hline & C.D@ 5\% & - & - & 0.09 & 0.15 & 0.37 & 0.24 & 0.26 & 5.56 \\
\hline
\end{tabular}


Abbey et al., (2000) and Leilah et al., (2003) reported that the difference in storability of genotypes may be due to the genetic variation. The respiration heat might be responsible for higher weight loss of onion bulbs in the storage (Moazam, 1983). From the study, the variety Bhima Shakthi was found to be more suitable for long term storage. The minimum storage losses in Bhima Shakthi was also due high TSS and high dry matter content of the bulbs as reported by Trivedi and Dhumal (2010).

\section{References}

Abbey, L., Danquah, O. A., Kanton, R. A. L. and Olympio, N. S., 2000. Characteristics and storage performance of eight onion cultivars. Ghana Journal of Science. 40: 9-13.

Brewster, J. L., 1990. Physiology of crop growth and bulbing. Onions and Allied Crops, pp.53-81.

Brewster, J. L., 1994. Onions and other vegetable Alliums. CAB Intl., Cambridge, England, pp.121-125.

CSIR., 2003. The Wealth of India: A dictionary of Indian raw materials and industrial products. Council of Science and Information Research: New Delhi. 1(1): 167-181.

Dhotre, M., 2009. Studies on genetic diversity and influence of nitrogen sources on performance of kharif onion (Allium cepa var. cepa L.). M. Sc. Thesis, University of Agricultural Sciences, Dharwad, India.

Jamali, L. A., Ibupoto, K. A., Chattha, S. H. and Laghari, R. B., 2012. Study on physiological weight loss in onion variety during storage. Pakistan Journal of Agricultural Sciences. 28(1): 1-7.

Kedar, A.A., Zagory, D. and Kerbal, E.L., 1989. Modified atmosphere packaging of fruits and vegetables. Critical Reviews in Food Science and Nutrition. 50: 761-766.
Kukanoor, L., 2005. Post-harvest studies in onion Cv. N-53. Ph. D. (Hort.) Thesis, University of Agricultural Sciences, Dharwad, India.

Leilah, A. A., El-Kalla, S.A., Mostafa, A.K. and Afifi, H.M.A., 2003. Performance of some local Egyptian onion strains under different planting dates. Scientific Journal of King Faisal University. 4 (1): 119-136.

Moazam, H., 1983. Influence of nitrogen fertilizer, irrigation and pre-harvest foliar application of maleic hydrazide on postharvest behaviour of onion (Allium cepa L.) bulbs under several storage conditions. Ph. D. Thesis, Mahatma Phule Krishi Vidyapeeth, Rahuri.

Rabinowitch, H. D. andCurrah, L., 2002. Evolution, domestication and taxonomy. In:(eds) Allium crop science: recent advances. CAB Int. Wallingford, UK, pp. 372-395.

Shanmugasundaram, S., 1999. Effect of bulb size and storage conditions on storability of onions. AVRDC report, p. 25.

Shanmugasundaram, S., 2000. Storage loss and disease incidence of 12 onion cultivars in ambient storage room 1 (SR1) and storage room 2 (SR2) after 15 weeks of storage, AVRDC report, p. 2627.

Shanmugasundaram, S., 2003. Development of onions with good storability. AVRDC report, p. 3-5.

Trivedi, A. P. and Dhumal, K. N., 2010. Variability and correlation studies on bulb yield, morphological and storage characters in onion (Allium cepa L.). Journal of Pure and Applied Sciences. 18: $1-4$.

Utagi, S., Anjanappa, M., Kale, S.M. and Badiger, M., 2015. Influence of different onioncultivars on storage life under central dry zone of Karnataka. International Journal of Processing and Post-harvest Technology. 6 (1): 36-40. 


\section{How to cite this article:}

Veena Goni, Devaraju, V. Srinivasa, D. Lakshmana and Kantharaj, Y. 2020. Influence of Different Onion Cultivars on Storage Life under Hill Zone of Karnataka. Int.J.Curr.Microbiol.App.Sci. 9(10): 3127-3133. doi: https://doi.org/10.20546/ijcmas.2020.910.375 